\title{
DETECTION OF VERY HIGH ENERGY RADIATION FROM THE BL LACERTAE OBJECT PG 1553+113 WITH THE MAGIC TELESCOPE
}

J. Albert, ${ }^{1}$ E. Aliu, ${ }^{2}$ H. Anderhub,${ }^{3}$ P. Antoranz, ${ }^{4}$ A. Armada, ${ }^{2}$ C. Baixeras, ${ }^{5}$ J. A. Barrio, ${ }^{4}$ H. Bartko, ${ }^{6}$ D. Bastieri, ${ }^{7}$ J. Becker,${ }^{8}$ W. Bednarek, ${ }^{9}$ K. Berger, ${ }^{1}$ C. Bigongiari ${ }^{7}$ A. Biland, ${ }^{3}$ R. K. Bock, ${ }^{6,7}$ P. Bordas, ${ }^{10}$ V. Bosch-Ramon, ${ }^{10}$

T. Bretz, ${ }^{1}$ I. Britvitch, ${ }^{3}$ M. Camara, ${ }^{4}$ E. Carmona, ${ }^{6}$ A. Chilingarian, ${ }^{11}$ S. Ciprini, ${ }^{12}$ J. A. Coarasa, ${ }^{6}$ S. Commichau, ${ }^{3}$

J. L. Contreras, ${ }^{4}$ J. Cortina, ${ }^{2}$ V. Curtef, ${ }^{8}$ V. Danielyan, ${ }^{11}$ F. Dazzi, ${ }^{7}$ A. De Angelis, ${ }^{13}$ R. De los Reyes, ${ }^{4}$

B. De Lotto, ${ }^{13}$ E. Domingo-Santamaría, ${ }^{2}$ D. Dorner, ${ }^{1}$ M. Doro, ${ }^{7}$ M. Errando, ${ }^{2}$ M. Fagiolini, ${ }^{14}$ D. Ferenc, ${ }^{15}$

E. Fernández, ${ }^{2}$ R. Firpo, ${ }^{2}$ J. Flix,${ }^{2}$ M. V. Fonseca, ${ }^{4}$ L. Font,${ }^{5}$ M. Fuchs, ${ }^{6}$ N. Galante, ${ }^{6}$ M. Garczarczyk, ${ }^{6}$

M. Gaug ${ }^{7}$ M. Giller, ${ }^{9}$ F. Goebel,${ }^{6}$ D. Hakobyan,${ }^{11}$ M. Hayashida, ${ }^{6}$ T. Hengstebeck, ${ }^{16}$ D. HöHne, ${ }^{1}$ J. Hose, ${ }^{6}$

C. C. Hsu, ${ }^{6}$ P. Jacon, ${ }^{9}$ T. Jogler,${ }^{6}$ O. KaleKin, ${ }^{16}$ R. Kosyra, ${ }^{6}$ D. Kranich,${ }^{3,17}$ R. Kritzer, ${ }^{1}$ A. Laille, ${ }^{15}$

P. Liebing, ${ }^{6}$ E. Lindfors, ${ }^{12}$ S. Lombardi, ${ }^{7}$ F. Longo, ${ }^{13}$ J. López, ${ }^{2}$ M. López, ${ }^{4}$ E. Lorenz,${ }^{3,6}$ P. Majumdar, ${ }^{6}$

G. Maneva, ${ }^{18}$ K. Mannheim, ${ }^{1}$ O. Mansutti,${ }^{13}$ M. Mariotti,${ }^{7}$ M. Martínez, ${ }^{2}$ D. Mazin, ${ }^{6}$ C. Merck, ${ }^{6}$ M. Meucci, ${ }^{14}$

M. Meyer, ${ }^{1}$ J. M. Miranda, ${ }^{4}$ R. Mirzoyan, ${ }^{6}$ S. Mizobuchi, ${ }^{6}$ A. Moralejo, ${ }^{2}$ K. Nilsson, ${ }^{12}$ J. Ninkovic, ${ }^{6}$

E. Oña-Wilhelmi, ${ }^{2}$ N. Otte, ${ }^{6}$ I. Oya, ${ }^{4}$ D. Paneque,${ }^{6}$ R. Paoletti,${ }^{14}$ J. M. Paredes, ${ }^{10}$ M. Pasanen, ${ }^{12}$ D. Pascoli, ${ }^{7}$

F. Pauss,${ }^{3}$ R. Pegna, ${ }^{14}$ M. Persic, ${ }^{13,19}$ L. Peruzzo, ${ }^{17}$ A. Piccioli,${ }^{14}$ M. Poller,${ }^{1}$ N. Puchades,${ }^{2}$ E. Prandini,${ }^{7}$

A. Raymers, ${ }^{11}$ W. Rhode, ${ }^{8}$ M. Ribó, ${ }^{10}$ J. Rico, ${ }^{2}$ M. Rissi, ${ }^{3}$ A. Robert, ${ }^{5}$ S. Rügamer, ${ }^{1}$ A. Saggion, ${ }^{7}$ A. Sánchez, ${ }^{5}$

P. Sartori, ${ }^{7}$ V. Scalzotto, ${ }^{7}$ V. Scapin, ${ }^{7}$ R. Schmitt, ${ }^{1}$ T. Schweizer, ${ }^{6}$ M. Shayduk, ${ }^{6,16}$ K. Shinozaki, ${ }^{6}$ N. Sidro, ${ }^{2}$

A. Sillanpä̈̈, ${ }^{12}$ D. Sobczynska, ${ }^{9}$ A. Stamerra, ${ }^{14}$ L. S. Stark, ${ }^{3}$ L. Takalo, ${ }^{12}$ P. Temnikov, ${ }^{18}$ D. Tescaro, ${ }^{2}$

M. Teshima, ${ }^{6}$ N. Tonello,${ }^{6}$ D. F. Torres, ${ }^{2}$ N. Turini, ${ }^{14}$ H. Vankov, ${ }^{18}$ V. Vitale, ${ }^{13}$

R. M. Wagner, ${ }^{6,17}$ T. Wibig, ${ }^{9}$ W. Wittek, ${ }^{6}$ R. ZAnin, ${ }^{7}$ And J. Zapatero ${ }^{5}$

Received 2006 May 25; accepted 2006 December 4; published 2006 December 27

\section{ABSTRACT}

In 2005 and 2006, the MAGIC telescope observed very high energy gamma-ray emission from the distant BL Lac object PG $1553+113$. The overall significance of the signal was $8.8 \sigma$ for $18.8 \mathrm{hr}$ of observation time. The light curve shows no significant flux variations on a daily timescale; the flux level during 2005 was, however, significantly higher compared to 2006. The differential energy spectrum between $\sim 90$ and $500 \mathrm{GeV}$ is well described by a power law with photon index $\Gamma=4.2 \pm 0.3$. The combined 2005 and 2006 energy spectrum provides an upper limit of $z=0.74$ on the redshift of the object.

Subject headings: BL Lacertae objects: individual (PG 1553+113) — gamma rays: observations

Online material: color figure

\section{INTRODUCTION}

\subsection{The BL Lac Object PG $1553+113$}

The active galactic nucleus (AGN) PG 1553+113 was first reported in the Palomar-Green catalog of UV-bright objects (Green et al. 1986). It was the only new BL Lac object found in the survey and the first BL Lac object found in an optical survey. Its spectrum is, typically for BL Lac objects, featureless

\footnotetext{
${ }^{1}$ Universität Würzburg, Germany.

${ }^{2}$ Institut de Física d'Altes Energies, Bellaterra (Barcelona), Spain.

${ }^{3}$ ETH Zurich, Switzerland.

${ }^{4}$ Universidad Complutense, Madrid, Spain.

${ }^{5}$ Universitat Autònoma de Barcelona, Bellaterra, Spain.

${ }^{6}$ Max-Planck-Institut für Physik, Munich, Germany.

${ }^{7}$ Università di Padova and INFN, Padova, Italy.

${ }^{8}$ Universität Dortmund, Germany.

${ }^{9}$ University of Łódź, Poland.

${ }^{10}$ Universitat de Barcelona, Spain.

${ }^{11}$ Yerevan Physics Institute, Yerevan, Armenia.

12 Tuorla Observatory, Turku University, Piikkiö, Finland.

${ }^{13}$ Università di Udine and INFN Trieste, Udine, Italy.

${ }^{14}$ Università di Siena and INFN Pisa, Siena, Italy.

${ }^{15}$ University of California, Davis, CA.

${ }^{16}$ Humboldt-Universität zu Berlin, Germany.

${ }^{17}$ Corresponding authors: D. Kranich (kranich@physics.ucdavis.edu) and

R. Wagner (rwagner@mppmu.mpg.de).

${ }^{18}$ Institute for Nuclear Research and Nuclear Energy, Sofia, Bulgaria.

${ }^{19}$ INAF-Osservatorio Astronomico and INFN, Trieste, Italy.
}

(Miller \& Green 1983), and the optical variability strong $\left(m_{p}=13.2-15.0\right.$; Miller et al. 1988). The spectral characteristics are close to those of X-ray-selected BL Lac objects (Falomo \& Treves 1990), and it is classified in the literature as an intermediate (Laurent-Muehleisen et al. 1999; Nieppola et al. 2006) or high-frequency peaked BL Lac object (Giommi et al. 1995), as its synchrotron peak frequency lies on the borderline of these two groups.

Despite several attempts, no emission or absorption lines have been found in the spectrum of PG 1553+113 (Falomo $\&$ Treves 1990). Thus only indirect methods can be used to determine the redshift $z$ (e.g., Sbarufatti et al. 2005, 2006). The host galaxy was not resolved in Hubble Space Telescope (HST) images (Urry et al. 2000); it is therefore safe to assume $z>$ 0.25 . The observation of very high energy (VHE, defined here as $E \gtrsim 100 \mathrm{GeV}) \gamma$-ray emission, on the other hand, may permit to set an upper limit on $z$. The $\gamma$-ray absorption in the extragalactic background light (EBL) by means of $e^{+}, e^{-}$pair production (Stecker et al. 1992; Aharonian et al. 2006a) can significantly affect the shape of the observed energy spectrum depending on the source redshift. Based on present-day EBL models and the observed $\gamma$-ray spectrum, one can derive the intrinsic spectrum as a function of $z$. Physical constraints on, e.g., the slope of the intrinsic spectrum may then permit the 


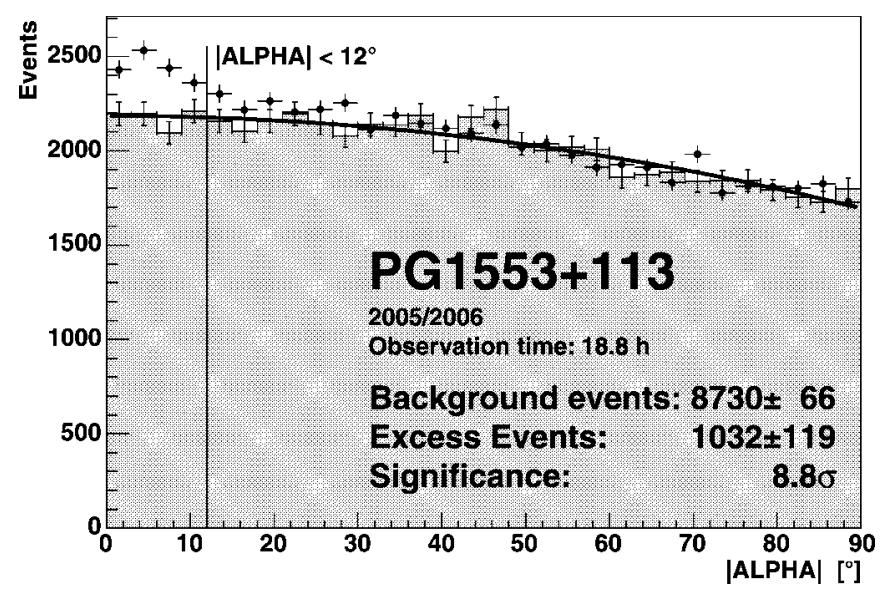

FIG. 1.-ALPHA plot for the combined 2005 and 2006 PG 1553+113 data after cuts. The diagram also shows the distribution of the (normalized) ofF data and a second-order polynomial describing the ofF data. [See the electronic edition of the Journal for a color version of this figure.]

setting of upper limits on the possible redshift (Aharonian et al. 2006b).

PG $1553+113$ belongs to a catalog of X-ray bright objects (Donato et al. 2005) and, based on its spectral energy distribution (SED) properties, was one of the most promising candidates from a list of VHE $\gamma$-ray emitting candidates proposed by Costamante \& Ghisellini (2002). So far, upper limits on the $\gamma$-ray emission have been reported by the Whipple collaboration (19\% Crab flux above $390 \mathrm{GeV}$; de la Calle Perez et al. 2003) and Milagro (Williams 2005). Recently the HESS collaboration has presented evidence for a $\gamma$-ray signal at the $4 \sigma$ level (up to $5.3 \sigma$ using a low energy threshold analysis) above $200 \mathrm{GeV}$ corresponding to about $2 \%$ of the Crab flux (Aharonian et al. 2006b). The energy spectrum was found to have a steep slope with $\Gamma=4.0 \pm 0.6_{\text {stat }}$, and an upper limit on the redshift of $z<0.74$ was derived.

\subsection{The MAGIC Telescope}

The MAGIC telescope is located on the Canary Island of La Palma (N28.75 $, \mathrm{W} 17.86^{\circ}, 2225 \mathrm{~m}$ above sea level). The telescope comprises a $17 \mathrm{~m}$ diameter tessellated, parabolic mirror with a total surface of $234 \mathrm{~m}^{2}$, a light-weight space frame made from carbon fiber-epoxy tubes, and a camera with 576 hemispherical photomultiplier tubes (PMT) with enhanced quantum efficiency (Paneque et al. 2004). The field of view of the camera is $3.5^{\circ}$ while the trigger area covers about $2.0^{\circ}$ in diameter. The fast PMT analog signals are routed via optical fibers to the DAQsystem electronics in the counting house $80 \mathrm{~m}$ away. The signals are digitized by dual range $300 \mathrm{MHz}$ FADCs. MAGIC can explore $\gamma$-rays at energies down to $50 \mathrm{GeV}$ (trigger threshold, depending on the zenith angle), critical for the observation of medium-redshift VHE sources with steeply falling spectra such as PG $1553+113$. The MAGIC telescope parameters and performance are described in more detail in Baixeras et al. (2004) and Cortina et al. (2005).

Simultaneous with MAGIC, optical observations were performed with the KVA telescope on La Palma, operated remotely from Tuorla Observatory. The main instrument is a $60 \mathrm{~cm}$ (f/15) Cassegrain telescope equipped with a CCD capable of polarimetric measurements. A $35 \mathrm{~cm}$ auxiliary telescope (f/11) is mounted on the same RA axis. This telescope is used for BVRI CCD photometry.

\section{OBSERVATION AND DATA ANALYSIS}

PG $1553+113$ was observed with the MAGIC telescope for $8.9 \mathrm{hr}$ in 2005 April and May, i.e., at about the same time when HESS also observed the source, and for $19 \mathrm{hr}$ from 2006 January to April. In addition to the observations with MAGIC and the $35 \mathrm{~cm}$ photometric telescope simultaneous data in Xrays were taken with the All Sky Monitor on board the RXTE satellite. ${ }^{20}$ Data taken during nonoptimal weather conditions or affected by hardware problems were excluded from the analysis. Also, only data taken at small zenith angles $\mathrm{ZA}<30^{\circ}$ (corresponding to a low energy threshold that is suitable for a steep energy spectrum) were retained, although measurements went up to $53^{\circ}$. After these selection cuts, 7.0 and $11.8 \mathrm{hr}$ of good data remained for 2005 and 2006, respectively. Given the mean ZA of $\sim 22^{\circ}, \gamma$-ray events above $\sim 90 \mathrm{GeV}$ have been used for the physics analysis.

In addition to the so-called oN data from PG $1553+113$, OFF data were taken on a nearby sky position where no $\gamma$-ray source is expected, but with comparable zenith-angle distribution and night-sky background light conditions. The off data are used to determine the background content in the signal region of the on data. This was done by means of a secondorder polynomial fit (without linear term) to the ALPHA distribution of the normalized OFF data. The normalization was done in the ALPHA region between $30^{\circ}$ and $90^{\circ}$ where no $\gamma$ ray events are expected. The ALPHA parameter describes the orientation of a shower image in the camera with respect to the camera center. Air showers that are aligned parallel to the telescope axis do have ALPHA values close to zero. In total, $14.5 \mathrm{hr}$ of OFF data (6.5 hr from 2005 and $8.0 \mathrm{hr}$ from 2006) have been used for the analysis. Since the two off samples were in good agreement we used the combined data to analyze the individual on data samples.

The data were analyzed using the standard MAGIC analysis programs for calibration, image cleaning, cut optimization, and energy reconstruction (Bretz et al. 2005; Gaug et al. 2005; Wagner et al. 2005). The primary method for discrimination between hadron- and $\gamma$-ray-induced showers is based on the random forest (RF) method (Breiman 2001; Bock et al. 2004), which was trained on OFF data and Monte Carlo (MC) generated $\gamma$-ray events. The significance of any excess was calculated according to equation (17) in Li \& Ma (1983), where the oNto-OFF ratio $\alpha$ was derived, taking into account the smaller error from the off-data fit. In addition to the cut optimization, the RF method was also used for the energy estimation based on the image parameters of a statistically independent $\mathrm{MC} \gamma$ ray sample. The average energy resolution obtained was $24 \%$ rms. All MC data used in this analysis were generated using CORSIKA, version 6.019 (Knapp \& Heck 2004; Majumdar et al. 2005).

\section{RESULTS}

Combining the data from 2005 and 2006, a very clear signal is seen in the image parameter ALPHA, as shown in Figure 1. Defining the signal region as ALPHA $<12^{\circ}$ (containing about $90 \%$ of the $\gamma$-ray events), an excess of 1032 over 8730 background events yields a total significance of $8.8 \sigma$. The individual results for the years 2005 and 2006 are listed separately in Table 1 . In both years the object was clearly detected with a significance $>6 \sigma$.

\footnotetext{
${ }^{20}$ These data are provided on the Web at http://heasarc.gsfc.nasa.gov/ xte_weather.
} 
TABLE 1

Results from the PG 1553+113 Analysis as Derived For 2005 AND 2006

\begin{tabular}{|c|c|c|c|c|c|c|c|c|}
\hline Year & $\begin{array}{l}\text { ON Time } \\
\text { (hr) }\end{array}$ & $N_{\mathrm{ON}}$ & $N_{\text {OFF }}$ & $N_{\text {excess }}$ & ON/OFF & $\sigma$ & $F(E>200 \mathrm{GeV})^{\mathrm{a}}$ & Photon Index \\
\hline 2005 & 7.0 & 3944 & $3501 \pm 26$ & $443 \pm 68$ & 0.20 & 6.7 & $2.0 \pm 0.6_{\text {stat }} \pm 0.6_{\text {sys }}$ & $4.31 \pm 0.45$ \\
\hline 2006 & 11.8 & 5815 & $5228 \pm 39$ & $588 \pm 86$ & 0.30 & 7.0 & $0.6 \pm 0.2_{\text {stat }} \pm 0.2_{\text {sys }}$ & $3.95 \pm 0.23$ \\
\hline $2005+2006$ & 18.8 & 9761 & $8730 \pm 66$ & $1032 \pm 119$ & 0.49 & 8.8 & $1.0 \pm 0.4_{\text {stat }} \pm 0.3_{\text {sys }}^{\text {sys }}$ & $4.21 \pm 0.25$ \\
\hline
\end{tabular}

${ }^{\mathrm{a}}$ Integral flux in units of $10^{-11} \mathrm{~cm}^{-2} \mathrm{~s}^{-1}$.

The $\gamma$-ray, X-ray, and optical light curves of PG $1553+113$ are shown in Figure 2. While the optical data show significant short-term variability on the $25 \%$ level, the X-ray data are consistent with a constant emission, given the weighted mean of $0.15 \pm 0.03$ counts $\mathrm{s}^{-1}$. In $\gamma$-rays there is no evidence for short-term variability, but a significant change in the flux level from 2005 to 2006 is found, given a systematic error of the analysis on the flux level of about $30 \%$. The average integral flux between 120 and $400 \mathrm{GeV}$ is given as $F=10.0 \pm$ $0.23_{\text {stat }}$ and $3.7 \pm 0.08_{\text {stat }}$ (with $F$ given in units of $10^{-11} \mathrm{~cm}^{-2}$ $\mathrm{s}^{-1}$ ) for 2005 and 2006, respectively. On February 25, prior to the optical flare, the optical polarimetry of the source was measured with the KVA $60 \mathrm{~cm}$ telescope. The degree of optical linear polarization was $8.3 \% \pm 0.2 \%$ and the polarization position angle was $139.1^{\circ} \pm 0.4^{\circ}$. It should be noted that since the host galaxy cannot be resolved for this object, the optical flux should correspond to the emission from the AGN core.

The combined 2005 and 2006 differential energy spectrum for PG $1553+113$ is shown in Figure 3. The integral fluxes above $200 \mathrm{GeV}$ and the spectral slope coefficients for the different samples are listed in Table 1. Effects on the spectrum determination introduced by the limited energy resolution were corrected by "unfolding" according to Mizobuchi et al. (2005). For comparison, the MAGIC Crab energy spectrum and the HESS PG 1553+113 energy spectrum (Aharonian et al. 2006b) are also shown. The energy spectrum is well described by a pure power law:

$$
\frac{d N}{d E}=\left(1.8 \pm 0.3_{\text {stat }}\right)\left(\frac{E}{200 \mathrm{GeV}}\right)^{-4.2 \pm 0.3_{\text {stat }}}
$$

(in units of $10^{-10} \mathrm{~cm}^{-2} \mathrm{~s}^{-1} \mathrm{TeV}^{-1}, \chi^{2} / \mathrm{dof}=1.5 / 4$ ). Compared to the Crab spectrum in the same energy range $(\Gamma=2.41 \pm$ 0.05 ; Wagner et al. 2005), this spectrum is significantly steeper. The spectral slopes of the individual years are in good agree-

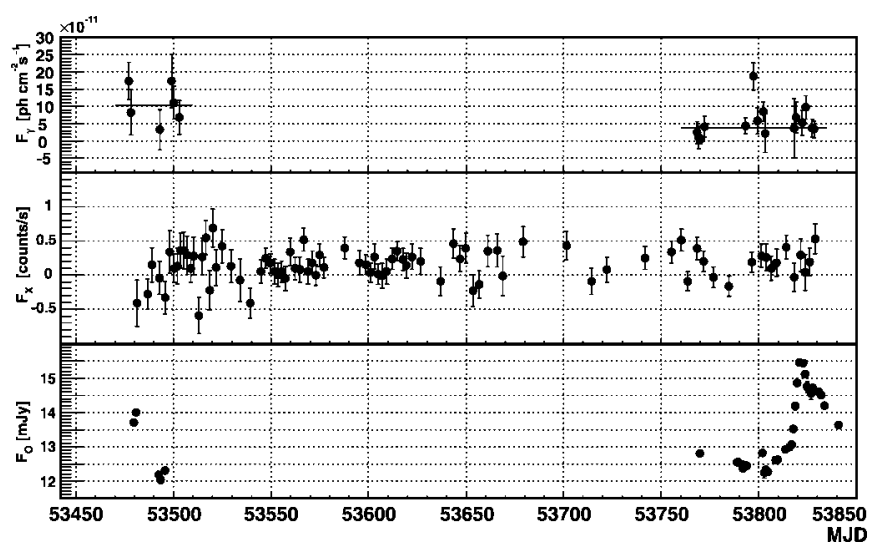

FIG. 2.-VHE $\gamma$-ray $(120-400 \mathrm{GeV})$, X-ray (2-10 keV), and optical light curve ( $R$ band) of PG $1553+113$ in 2005 and 2006. The horizontal bars in the top panel correspond to the average flux during 2005 and 2006. ment, although the flux level above $200 \mathrm{GeV}$ is about a factor 3 larger in 2005 compared to 2006 . This is also shown in the light curve. The estimated systematic error on the analysis (signal extraction, cut efficiencies, etc.) is 25\% (Fig. 3, dark colored band) and $30 \%$ on the energy scale (light colored band).

\section{DISCUSSION}

The BL Lac object PG $1553+113$ was detected at $8.8 \sigma$ with the MAGIC telescope in $18.8 \mathrm{hr}$ of observation in 2005 and 2006. This confirms the tentative signal seen by HESS at a higher energy threshold with data taken at about the same time as MAGIC in the 2005 period (Aharonian et al. 2006b). The source, therefore, can now be considered as firmly detected.

The agreement between the measured HESS and MAGIC energy spectra of PG $1553+113$ in 2005 is reasonably good. While the spectral slope is consistent within errors, the absolute flux above $200 \mathrm{GeV}$ in 2005 is a factor of 4 larger than with HESS. This difference may in part be explained by the systematic errors of both measurements, but also by variations in the flux level of the source (the observations with HESS were commenced after those with MAGIC). The observed energy spectrum is steeper than that of any other known BL Lac object. This may be an indication of a large redshift $(z \gtrsim 0.3)$, but can as well be attributed to intrinsic absorption at the AGN or, more naturally, to an inverse Compton peak position at lower energies. The spectrum can, however, be used to derive an upper limit on the source redshift from physical constrains on the intrinsic photon index $\left(\Gamma_{\mathrm{int}}>1.5\right)$, as discussed in Aharonian et al. (2006b). Using the lower limit on the evolving EBL density from Kneiske et al. (2004), we derived a $2 \sigma$ upper limit on the redshift of $z<0.74$. The same value was reported by Aharonian et al. (2006b), where a slightly different EBL model was used.

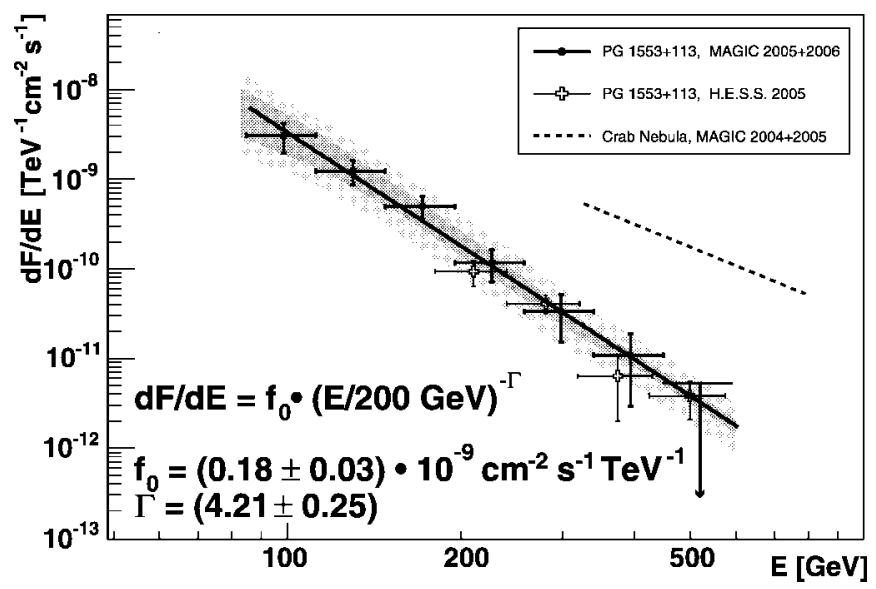

FIG. 3.-Differential energy spectrum of PG $1553+113$ as derived from the combined 2005 and 2006 data. The MAGIC Crab energy spectrum and the HESS PG 1553+113 energy spectrum have been included for comparison. 
The broadband SED of PG $1553+113$, together with the results from a model calculation, is shown in Figure 4 . The VHE data points correspond to the intrinsic spectrum of PG $1553+113$ as derived for a redshift of $z=0.3$. The black points at low energies denote the average optical and X-ray flux taken at the same time as the MAGIC observations. The gray hatched radio, optical, and X-ray nonsimultaneous data are taken from Giommi et al. (2002). The solid line shows the result of a model fit to the simultaneously recorded data (black points) using a homogeneous, one-zone synchrotron self-Compton (SSC) model as provided by Krawczynski et al. (2004). As can be seen from Figure 4 , the $\gamma$-ray, X-ray, and optical data are well described by the model. This is not the case for the radio data, where intrinsic absorption requires a much larger emitting volume compared to X-rays and $\gamma$-rays. Except for a somewhat smaller radius of the emitting region, model parameters identical to those in Costamante \& Ghisellini (2002) have been used: Doppler factor $D=21$, magnetic field strength $B=$ $0.7 \mathrm{G}$, radius of the emitting region $R=1.16_{-0.21}^{+0.62} \times 10^{16} \mathrm{~cm}$, electron energy density $\rho_{e}=0.11_{-0.06}^{+0.18} \mathrm{ergs} \mathrm{cm}^{-3}$, and slope of the electron distribution $\alpha_{e}=-2.6$ for $8.2<\log (E / \mathrm{eV})<$ $9.8_{-0.05}^{+0.2}$ and $\alpha_{e}=-3.6$ for $9.8_{-0.05}^{+0.2}<\log (E / \mathrm{eV})<10.6_{-0.0}^{+1.6}$. The limits on some of these parameters indicate the change of the SED model parameters when varying the assumed redshift from $z=0.2$ up to $z=0.7$ (parameters without limits were kept constant for all fits). In the case of $z \geq 0.56$ the SED model cannot accurately describe the data, and based on the obtained $\chi^{2}$ value, a redshift of 0.56 is excluded on the $4.5 \sigma$ level. For a comparison of the model parameters with those from other BL Lac objects we refer the reader to Costamante \& Ghisellini (2002).

PG $1553+113$ was in a high state in the optical in both years, showing a strong flare at the end of 2006 March. The high linear polarization of the optical emission $(8.3 \% \pm$ $0.2 \%$ ) indicates that a sizeable fraction of the optical flux is indeed synchrotron radiation. In $\gamma$-rays only a significant change in the flux level from 2005 to 2006 is found, while

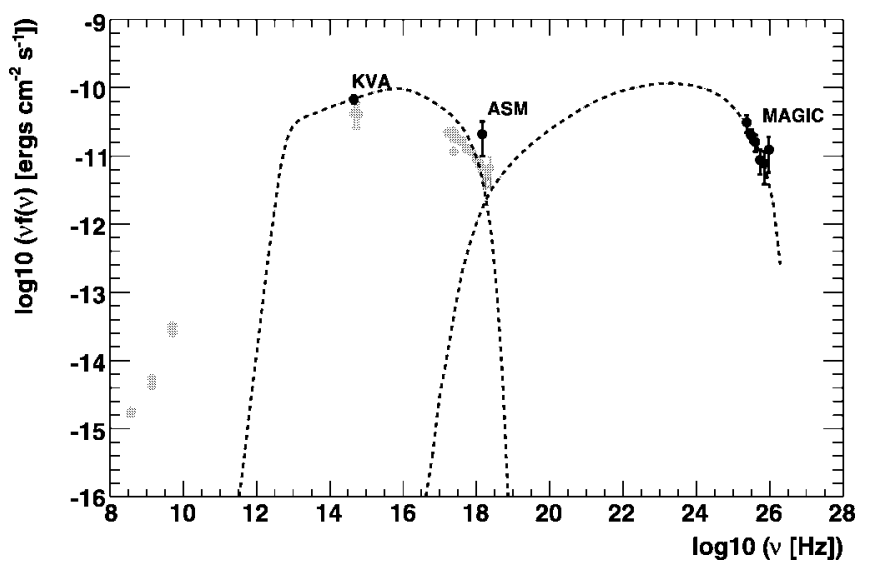

FIG. 4.-Broadband SED of PG $1553+113$. The solid lines are the result of a SSC model fit to the black data points using the code provided by Krawczynski et al. (2004) (see text). The gray points comprise nonsimultaneous radio, optical and X-ray data from Giommi et al. (2002).

there is no evidence for variability in X-rays. As a result, a possible correlation between the different energy bands cannot be established. A possible connection between the $\gamma$-ray detection and the optical high state can, however, not be excluded. The optical flare without an X-ray or $\gamma$-ray counterpart may still be explained by external inverse Compton (EIC) models that predict a time lag of the X-rays and $\gamma$-rays with respect to the optical emission.

We would like to thank the IAC for the excellent working conditions at the La Palma Observatory at Roque de los $\mathrm{Mu}-$ chachos. We are grateful to the RXTE ASM team for their quick-look results. The support of the German BMBF and MPG, the Italian INFN, and the Spanish CICYT is gratefully acknowledged. This work was also supported by ETH research grant TH-34/04-3 and by Polish grant MNiI 1P03D01028.

\section{REFERENCES}

Aharonian, F. et al. 2006a, Nature, 440, 1018 2006b, A\&A, 448, L19

Baixeras, C., et al. 2004, Nucl. Instrum. Methods Phys. Res. A, 518, 188 Bock, R. K., et al. 2004, Nucl. Instrum. Methods Phys. Res. A, 516, 511 Breiman, L. 2001, Machine Learning, 45, 5

Bretz, T., et al. 2005, in AIP Conf. Proc. 745, High-Energy Gamma-Ray Astronomy, ed. F. A. Aharonian, H. J. Völk, \& D. Horns (New York: AIP), 730

Cortina, J., et al. 2005, preprint (astro-ph/0508274)

Costamante, L., \& Ghisellini, G. 2002, A\&A, 384, 56

de la Calle Perez, I., et al. 2003, ApJ, 599, 909

Donato, D., Sambruna, R. M., \& Gliozzi, M. 2005, A\&A, 433, 1163

Falomo, R., \& Treves, A. 1990, PASP, 102, 1120

Gaug, M., et al. 2005, preprint (astro-ph/0508274)

Giommi, P., Ansari, S. G., \& Micol, A. 1995, A\&AS, 109, 267

Giommi, P., Massaro, E., \& Palumbo, G. 2002, in Proc. Blazar Astrophysics with BeppoSAX and other Observatories, ed. P. Giommi, E. Massaro, \& G. G. C. Palumbo (Frascati: ESA-ESRIN), 63

Green, R. F., Schmidt, M., \& Liebert, J. 1986, ApJS, 61, 305

Knapp, J., \& Heck, D. 2004, EAS Simulation with CORSIKA: A User's Guide (Karlsruhe: Inst. Kernphysik)

Kneiske, T. M., Bretz, T., Mannheim, K., \& Hartmann, D. H. 2004, A\&A, 413, 807
Krawczynski, H., et al. 2004, ApJ, 601, 151

Laurent-Muehleisen, S. A., Kollgaard, R. I., Feigelson, E. D., Brinkmann, W., \& Siebert, J. 1999, ApJ, 525, 127

Li, T., \& Ma, Y. 1983, ApJ, 272, 317

Majumdar, P., et al. 2005, preprint (astro-ph/0508274)

Miller, H. R., Carini, M. T., Gaston, B. J., \& Hutter, D. J. 1988, A Decade of UV Astronomy with the IUE Satellite (Paris: ESA), 303

Miller, H. R., \& Green, R. F. 1983, BAAS, 15, 957

Mizobuchi, S., et al. 2005, preprint (astro-ph/0508274)

Nieppola, E., Tornikoski, M., \& Valtaoja, E. 2006, A\&A, 445, 441

Paneque, D., Gebauer, H. J., Lorenz, E., \& Mirzoyan, R. 2004, Nucl. Instrum. Methods Phys. Res. A, 518, 619

Sbarufatti, B., Treves, A., \& Falomo, R. 2005, ApJ, 635, 173

Sbarufatti, B., Treves, A., Falomo, R., Heidt, J., Kotilainen, J., \& Scarpa, R. 2006, AJ, 132,

Stecker, F. W., de Jager, O. C., \& Salamon, M. H. 1992, ApJ, 390, L49

Urry, C. M., Scarpa, R., O’Dowd, M., Falomo, R., Pesce, J. E., \& Treves, A. 2000, ApJ, 532, 816

Wagner, R. M. et al. 2005, preprint (astro-ph/0508244)

Williams, D. 2005, in AIP Conf. Proc. 745, High-Energy Gamma-Ray Astronomy, ed. F. A. Aharonian, H. J. Völk, \& D. Horns (New York: AIP), 499 